\title{
Atmósferas afectivas del postconflicto en Colombia: narrativas de ciudadanos colombianos residentes en Quito, Ecuador
}

Jesús David Salas Betin

\section{Resumen}

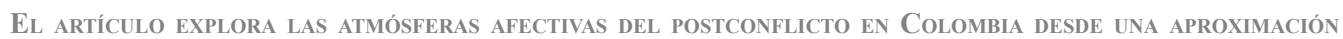
DESCRIPTIVA a PARTIR de datos CUANTITATIVOS, EVIDENCIADAS EN UN GRUPO DE NARRATIVAS DE CIUDADANOS colombianos residentes en la CiUdad de Quito, Ecuador. El concepto de atmósferas afectivas, acuñado POR BEN ANDERSON (2009), HACE REFERENCIA A LA INTERACCIÓN DE LA SUBJETIVIDAD INDIVIDUAL Y LOS ELEMENTOS MATERIALES QUE ASOCIAN LAS EMOCIONES CON EL AFECTO. LOS INSUMOS PARA EL ANÁLISIS SON PRODUCTO DEL proyecto En Modo P: EN Modo Persona, EN Modo Pos, que recoge cientos de voces de colombianos que, en un minuto, cuentan cómo el acuerdo de paz con las Fuerzas Armadas Revolucionarias de Colombia (FARC) HA AFECTAdO SU VIDA COTIDIANA. LOS RESULTADOS PRELIMINARES APUNTAN A REVELAR QUE LOS AFECTOS Y LAS EMOCIONES CONSTRUYEN REALIDAD SOCIAL Y, A PARTIR DE ELLOS, LAS PERSONAS CONSTRUYEN EL SIGNIFICADO SOCIAL DE SUS INSTITUCIONES.

Palabras Clave: teoría del afecto; atmósferas afectivas; postconflicto; Colombia.

Affective Atmospheres of Post-conflict in Colombia: Colombian RESIDENTS' nARRATIVES LIVING IN QUito

\begin{abstract}

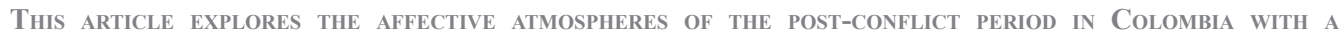
DESCRIPTIVE APPROACH BASED ON QUANTITATIVE DATA, EVIDENCED IN A GROUP OF NARRATIVES OF COLOMBIAN CITIZENS LiVING IN Quito, ECUADOR. THE CONCEPT OF AFFECTIVE ATMOSPHERES, COINED BY BEN ANDERSON (2009), REFERS TO THE INTERACTION OF INDIVIDUAL SUBJECTIVITY WITH THE MATERIAL ELEMENTS THAT ASSOCIATE EMOTIONS WITH affection. The inputs for the analysis are a product of the project "In Mode P: In Person Mode, In Pos Mode", a COllection of hundreds of Voices of Colombians who, in one MinUte, tell how the Peace agreement with the Revolutionary Armed Forces of Colombia (FA RC) has afFected their daily life. THE PRELIMINARY RESULTS POINT TO REVEALING THAT AFFECTIONS AND EMOTIONS BUILD SOCIAL REALITY, AND THAT PEOPLE CONSTRUCT THE SOCIAL MEANING OF THEIR INSTITUTIONS FROM THEM.
\end{abstract}

KeYwORdS: AfFeCt THEORY - AFFECTIVE ATMOSPHERES - POST-CONFLICT - COLOMBIA.

* Sociólogo de la Universidad Nacional de Colombia. Maestro en Sociología de la Facultad Latinoamericana de Ciencias Sociales, FLACSO Ecuador. Personal docente del Instituto de Altos Estudios Nacionales, IAEN. Correo electrónico: jesus.salas@iaen.edu.ec. 


\section{Introducción}

D 124 de noviembre de 2016, el gobierno colombiano, liderado por Juan Manuel Santos, y las Fuerzas Armadas Revolucionarias de Colombia (FARC), firmaron el acuerdo para la terminación definitiva del conflicto. La importancia de este acontecimiento radica en el fin de una de las guerras más largas de Latinoamérica y el mundo, que causó, al menos 8.299.334 víctimas, de acuerdo con datos de la Unidad para la Atención y Reparación Integral a las Victimas de Colombia (UARIV). Esto es, el 17\% del total de la población del país (Codhes, 2018).

Como era de esperarse, un acontecimiento de esta naturaleza acaparó la atención mundial. Los organismos multilaterales y la cooperación internacional anunciaron ayudas económicas para financiar los costos de la reconstrucción del tejido político y social desde antes de que se firmara el acuerdo. Los medios nacionales e internacionales volcaron su atención a cada uno de los momentos del proceso final. La agenda pública del gobierno de turno dio prioridad a la implementación de medidas que garantizaran el normal desarrollo del proceso. La academia introdujo en su vocabulario nuevos conceptos y empezó a pensarse la sociedad del postacuerdo. La gente, desde sus espacios cotidianos, empezó a debatir alrededor de las expectativas de este acontecimiento.

Fue en este marco que surgió el proyecto Modo P: En Modo Persona, En Modo Pos, como una iniciativa académico-periodística que buscaba capturar la esencia emocional del momento. Tomando como punto de partida la Teoría de los Afectos, se planteó como hipótesis el hecho de que los afectos y las emociones producen realidad social. Con el apoyo de OXFAM Internacional, LaSillaVacia.com y FLACSO Ecuador, reunió a un equipo de trabajo interdisciplinario con un objetivo en común: construir una narrativa que recogiera el mayor número de voces diversas posibles sobre el impacto de la firma de la paz con las FARC en la vida cotidiana de las personas y los sentimientos asociados a este hecho. Este artículo recoge los resultados preliminares de ese proceso.

El artículo está dividido en cinco partes. En la primera parte, se revisa brevemente los principales postulados del giro afectivo en las ciencias sociales y la noción de atmósferas afectivas, como elementos principales del marco teórico que cobija el proyecto. En la segunda parte, se busca familiarizar al lector con los detalles del proyecto En Modo P: En Modo Persona, En Modo Pos, el contexto social del acuerdo de paz con las FARC y la situación migratoria de la población colombiana residente en Quito. En la tercera parte, se presenta el detalle metodológico del proyecto y la información del trabajo de campo realizado en la ciudad de Quito. En la cuarta parte, se exponen los resultados preliminares, a partir de un análisis descriptivo de los datos obtenidos en el trabajo de campo. En la última parte, se discuten los resultados atendiendo a los escenarios del acuerdo final, las emocionalidades que genera y las atmósferas afectivas que se pueden identificar preliminarmente.

\section{Marco teórico}

En las últimas tres décadas, las ciencias sociales han visto el nacimiento de nuevas perspectivas analíticas que confluyen en nuevas teorías, donde cada vez más los sensitivo y lo emocional buscan explicar la realidad social. Estas perspectivas teóricas, no solo buscan desestructurar las formas clásicas de abordar y entender los problemas sociales, sino que se constituyen en espacios de confluencias para las diversas disciplinas, propiciando el dialogo de saberes entre las distintas ramas de las ciencias sociales. Establecen, pues, verdaderos giros en la forma de pensar y percibir la realidad, constituyendo nuevos marcos epistemológicos y metodológicos que desafían las formas tradicionales de investigar. 


\section{El giro afectivo en las ciencias sociales}

El giro afectivo constituye una de esas perspectivas teóricas que iluminan las nuevas formas de entender y percibir la realidad social. El desafío que se plantea esta perspectiva radica en lograr una síntesis entre lo sensitivo, referido a lo corporal, y lo emocional, como reflejo de la mente. De acuerdo con Hardt (2007), este desafío se fundamenta en dos aspectos. En primer lugar, porque el afecto refiere por igual al cuerpo y la mente; en segundo lugar, porque involucra la razón y la pasión. Por su parte, Lara y Enciso-Domínguez (2013: 101), indican que el giro afectivo atiende a "dos urgencias teóricas: el interés en la emocionalización de la vida pública, y el esfuerzo por reconfigurar la producción de conocimiento encaminado a profundizar en dicha emocionalización". En efecto, el giro afectivo combina, entre otros, postulados de la teoría psicoanalítica, la teoría del actor red, los estudios feministas, la geografía cultural y las teorías posestructuralistas, para entender 'aquello que se siente' como una forma de explicar el poder de afectar nuestra realidad y, a su vez, el poder que tiene ella de afectarnos.

En palabras de Macón (2013: 9), "el giro afectivo puede ser entonces presentado como un proyecto destinado a indagar en formas alternativas de aproximarse a la dimensión afectiva, pasional o emocional -y discutir las diferencias que pueda haber entre estas tres denominacionesa partir de su rol en el ámbito público". Hace referencia a la diversidad léxica que supone el giro y que ha centrado el debate en la forma de definir su objeto de estudio. La tensión teórica de fondo es la relación entre el cuerpo y la mente, esto es, entre la materialidad corpórea y la experiencia subjetiva del significado. En esa disyuntiva, el afecto ha sido comúnmente vinculado a lo corpóreo, lo preconsciente, el lugar en donde cobra materialidad y corporeidad la experiencia subjetiva. Entre tanto, la emoción ha sido pensada como una interpretación individual del afecto asociada al proceso cognitivo que permite la comprensión del mundo (Lara y Enciso-Domínguez, 2013). Otros términos como la pasión y el sentimiento han sido subsumidos por el afecto y las emociones, pues, como menciona Macón (2013), al referir la pasividad de las emociones, son considerados parciales e insuficientes por algunos autores que se inscriben dentro de la perspectiva del giro.

\section{Afectos, emociones y atmósferas en la teoría de Ben Anderson}

El giro afectivo tiene múltiples corrientes que se configuran a partir de diversas fuentes (Baruch Spinoza, Chantal Mouffe, Gilles Deleuze y Félix Guattari, Jean Laplanche y JeanBertrand Pontalis, Alfred Whitehead). Aunque la tensión entre afecto y emoción parezca una simple diferencia semántica, lo cierto es que detrás de ella se esconde un conflicto mayor en relación con la comprensión del término afecto como estandarte del giro (Lara y EncisoDomínguez, 2013). La discusión de fondo es sobre la forma de captar la experiencia sensible. Autores como Hardt (2007) y Thrift (2008) resaltan la multiplicidad teórica del afecto y su articulación con las complejidades, reconfiguraciones y articulaciones con el poder.

Un intento loable por reconciliar ambos términos (afecto y emoción) a través de la creación de un nuevo vocabulario que permita recoger elementos opuestos, pero íntimamente asociados, es la noción de "atmósferas afectivas" propuesta por Ben Anderson (2009). Con este término el autor pretende comprender aspectos ambivalentes en la tensión existente entre afecto y emoción como la presencia y la ausencia, la materialidad y la idealidad, lo definido y lo indefinido, la singularidad y la generalidad, pues, como menciona Lara y Enciso-Domínguez (2013:111):

"Implican la interacción de la subjetividad individual asociada a la emoción y los elementos materiales y agentes de significados frecuentemente coludidos con el afecto. Para Ben Anderson, la distinción entre afecto y emoción pierde sentido, en la propuesta de las atmósferas afectivas" encontramos una preocupación por rebasar los límites de la militancia teórica en pro de una comprensión más amplia del fenómeno afectivo". 
El concepto de atmósferas es retomado por Anderson de la geografía cultural, especialmente del trabajo de Mikel Dufrenne sobre La Fenomenología de la Experiencia Estética (The Phenomenology of Aesthetic Experience), publicado originalmente en 1953. Allí, Dufrenne acude al dinamismo de las atmósferas como parte de un intento de distinguir los objetos estéticos de otros tipos de objetos. Las atmósferas le ayudan a Dufrenne a explicar cómo el mundo expresado desborda el contenido representativo de los objetos estéticos como una cualidad que no se puede traducir a través de las palabras, pero que comunican en sí mismas sentimientos (Anderson, 2009). Lo que le interesa resaltar a Anderson, y que resulta interesante para el objetivo del proyecto que motiva este artículo, es la calidad inacabada de las atmósferas afectivas, que se están formando y deformando constantemente, apareciendo y desapareciendo a media que los cuerpos entran en contacto entre sí.

\section{Contexto}

El 2016 fue un año trascendental para el conflicto armado colombiano. Después de más de 50 años de guerra, el gobierno nacional y la guerrilla de las FARC, una de las más antiguas de Latinoamericana, por fin llegaron a un acuerdo que busca poner fin a uno de los conflictos más viejos de la región. El acuerdo, cuya versión final fue firmada el 24 de noviembre se construyó en 3 fases, en las que no solo participaron presentantes políticos y militares de los actores en conflicto, sino que incluyó representantes de la sociedad civil, los gremios productivos y representantes de las víctimas. El documento final gira alrededor de 6 temas fundamentales para la terminación del conflicto y la construcción de una paz estable y duradera, a saber: (1) reforma rural integral, (2) participación política, (3) fin del conflicto, (4) solución al problema de drogas, (5) víctimas y, por último, (6) implementación, verificación y refrendación (OACP, 2016).

Si bien el acuerdo alcanzado entre las FARC y el gobierno marca un precedente histórico a nivel mundial, su implementación constituye el primer paso en la construcción del postconflicto. El acuerdo crea las condiciones iniciales para desencadenar un proceso progresivo de terminación del conflicto (Codhes, 2018). Quizá, el capítulo sobre víctimas sea uno de los más reveladores, pues, además de incorporar figuras clásicas de esclarecimiento de la verdad y medidas para la reparación, busca generar las garantías jurídicas para la satisfacción de sus derechos y la no repetición. En efecto, la participación de las víctimas no solo fue importante durante la fase de negociación del fin del conflicto, llevada a cabo entre octubre de 2012 y agosto de 2016, sino que constituye en el eje articulador del Sistema Integral de Verdad, Justicia, Reparación y No Repetición, a través del cual se espera integrar a la vida civil a los miembros de las FARC (OACP, 2016). No es para menos. Hasta el año 2016, la Unidad para la Atención y Reparación Integral a las Victimas registraba 8.299.334 de colombianos víctimas del conflicto armado y la violencia política en el país (Codhes, 2018). En tanto, el número colombianos en necesidad o condición de protección internacional registrado por el Alto Comisionado de las Naciones Unidas para los Refugiados (Acnur) hasta el 2015 alcanza las 340.240 personas (Codhes, 2018). Esta cifra, que más que un dato exacto constituye un estimativo, podría ser mayor si consideramos que "en Colombia es permanente la salida de exiliados, pero por lo regular no se autorreconocen como tales, porque no tienen conocimiento de la existencia de ese estatus legal o de las formas de protección para estos casos" (RincónSuarez, 2018, p.169).

\section{La migración de colombianos en Quito}

Se estima que el número de colombianos con estatus de residente en Ecuador supera las 120 mil personas, de las cuales 57 mil son reconocidas legalmente como refugiados, Quito es uno de los principales lugares de acogida (Codhes, 2018). Solanda, La Roldós, Carcelén, 
Carapungo, Lucha de Los Pobres, Centro Histórico y Comité del Pueblo son, quizás, los sectores con mayor presencia de colombianos en la ciudad. Estos barrios cumplen un conjunto de condiciones ideales para la llegada de la población colombiana que encuentra en Quito un lugar tranquilo para empezar una nueva vida fuera de su país de origen. Entre otras, se caracterizan por ser espacios periurbanos lo suficientemente cerca del epicentro de la ciudad como para facilitar su movilidad e inserción laboral; la presencia de otros colombianos ya establecidos, lo que posibilita la conformación de comunidades de connacionales; precios de los arriendos accesibles; entre otras (Ortega y Riaño-Alcalá, 2007). Además, estos barrios son señalados tradicionalmente como espacio de reivindicaciones sociales. Detrás de sus habitantes perviven historias de migración que, como el caso de muchos migrantes colombianos, llegaron a quito buscando mejores oportunidades de vida. Las condiciones de inserción en la vida cotidiana de la ciudad, bien sea desde el comercio informal o como asalariados de las fábricas que circundan estos sectores, posibilitó el surgimiento de formas organizativas propias alrededor del espacio público, el acceso a los servicios básicos, entre otras. Esta concepción aún pervive en la referencia espacial de muchos habitantes de Quito, que miran estos sectores como espacios de organización y demandas sociales.

\section{En Modo P: En Modo Persona, En Modo Pos}

El proyecto En Modo $P$ es una iniciativa de narrativa transmedia (Sánchez-Mesa, 2016) codirigido por Olga Lucia Lozano ${ }^{1}$ y Susana Wappenstein ${ }^{2}$. El proyecto se apoya en la teoría de los afectos para construir una narrativa incluyente a partir de los sentimientos que despierta cada etapa o acontecimiento del proceso de paz entre el gobierno de Colombia y las FARC (Salas, 2017). La idea surgió a partir de una particular forma de entender la teoría de los afectos aplicada al campo de la investigación sociológica, con la intención de generar narrativas diversas en las que la vida y los hechos claves asociados a la firma del acuerdo de paz fueran contados por el mayor número de voces posibles, abarcando un amplio espectro de miradas, lenguajes, perspectivas y emocionalidades (Arboleda, 2016).

Pero la intencionalidad del proyecto transciende el campo académico, pues en su génesis, fue concebido como "una instalación sonora que mezcla criterios de reportería y edición periodística, elementos de metodologías de análisis sociológicos desde la teoría de los afectos y el análisis de discurso, experimentación en formatos sonoros y narrativas artísticas" (Arboleda, 2016). De acuerdo con sus directoras, la idea que lo guía es la construcción de "la primera memoria emocional de la violencia en Colombia" (León, 2017). Para ello, cuenta con centenares de voces que, al fervor del momento, cuentan sus experiencias de vida alrededor del conflicto y el postconflicto. Las voces fueron almacenadas en una base de datos de acceso público, en la que las personas pueden interactuar a través de notas de audio. A su vez, los audios fueron clasificados en 12 sentimientos, cada uno de ellos simulando un sonido de la escala musical, pues, una de las intenciones del proyecto era realizar una composición musical que recogiera la esencia emocional del proceso.

En suma, el proyecto reconstruye una crónica que narra la vida cotidiana de habitantes de zonas como Sumapaz (Bogotá, Colombia), de la población colombiana migrante y en condición de refugio en Quito (Ecuador), de mujeres, niños, campesinos, grupos indígenas, estudiantes, empleados, y todo aquel que quisiera sumar su testimonio como parte de un proceso innovador de construcción de memoria colectiva ${ }^{3}$.

1 Periodista, cofundadora y ex editora creativa de La Silla Vacía.

2 Profesora del Departamento de Sociología y Estudios de Género en FLACSO, Ecuador.

3 Para consultar la base de datos y conocer más detalles del proyecto se puede consultar: http://enmodop.com/. 


\section{Metodología}

La metodología adoptada para capturar la información fue la entrevista. La estrategia utilizada fue el abordaje. En distintos momentos del proceso, se abordó a las personas en calles, manifestaciones ciudadanas, eventos académicos y homenajes a la memoria (como la semana por la paz) en el que confluyera la población colombiana en Quito para que, en máximo un minuto, respondiera dos preguntas, a saber: ¿qué impacto ha tenido en su vida cotidiana la firma de paz con las FARC?, y, ¿qué siente ahora con respecto a este acuerdo? Las respuestas de las personas fueron clasificadas en 12 categorías emocionales, de acuerdo con la emoción predominante en su relato. Las categorías emocionales y las emociones asociadas se presentan en la Tabla 1.

\section{Tabla 1. Categorías emocionales}

\begin{tabular}{|l|l|}
\hline Categoría emocional & Emociones asociadas \\
\hline Injusticia & mal, insatisfacción, pesimismo, impotencia, desilusión, desazón \\
\hline Indignación & furia, enojo, frustración, enfado, ira, irritación, disgusto \\
\hline Miedo & depresión, pena, desesperanza, inseguridad \\
\hline Conmoción & sorpresa, shock, desconcierto, consternación \\
\hline Incertidumbre & duda, preocupación, intriga \\
\hline Bien & conformidad, indiferencia, conveniencia \\
\hline Nostalgia & melancolía, tristeza, soledad, esperanza \\
\hline Calma & paz, tranquilidad, serenidad \\
\hline Satisfacción & apoyo, solidaridad, agradecimiento, equidad, justicia \\
\hline Esperanza & expectativa, optimismo, empoderamiento, motivación \\
\hline Felicidad & emoción, fascinación, excitación \\
\hline Euforia & alcha, entusiasmo \\
\hline
\end{tabular}

Fuente: En Modo P. Elaboración propia

En total, se realizaron 152 entrevistas entre septiembre de 2016 y marzo de 2017 en la ciudad de Quito. Las entrevistas, que se realizaron en distintos espacios de la ciudad, iban dirigidas a personas de nacionalidad colombiana residentes en Ecuador. La Tabla 2 muestra el número total de entrevistas desagregado por el año y el mes en el que se capturó la información. 


\begin{tabular}{|} 
TABla 2. Distribución De entrevistas POR AÑos Y MeS \\
\hline \multirow{2}{*}{ Año } & Mes & Total \\
\hline \multirow{2}{*}{2016} & Septiembre & 53 \\
\cline { 2 - 3 } & Octubre & 60 \\
\cline { 2 - 3 } & Noviembre & 17 \\
\cline { 2 - 3 } & Diciembre & 8 \\
\hline \multirow{2}{*}{2017} & Febrero & 6 \\
\hline & Marzo & 8 \\
\hline
\end{tabular}

Fuente: En Modo P. Elaboración propia

Al inicio de las entrevistas, se pidió a los entrevistados información personal para caracterizar sus testimonios en la plataforma. Estos datos, a su vez, servirían como variables de control para el análisis de la información recolectada. Vale decir que, la información otorgada por las personas era de carácter voluntario, por tanto, cuenta con el debido consentimiento informado. La información que se solicitó corresponde a: edad, género y lugar de origen.

La edad de los entrevistados se agrupó para tener datos más consistentes y facilitar la comparación al momento del análisis. La agrupación se realizó siguiendo los patrones generacionales de las personas entrevistadas y los ciclos de la violencia en Colombia. De esta forma, el primer grupo se encuentra conformado por menores de 18 años, cuyo testimonio se recolectó con el consentimiento de los padres, con la intención de entender la percepción histórica del conflicto y las consecuencias del acuerdo en las llamadas "nuevas generaciones". El segundo grupo lo conforman personas entre 18 y 34 años, es decir, personas nacidas en las décadas de 1980 y 1990 y, por tanto, que vivieron conscientemente el último periodo de violencia, iniciado en la década de los 90's. El tercer grupo corresponde a personas entre 35 y 55 años, esto es, personas nacidas en la década de 1960 y 1970 que, por tanto, vivieron de manera consciente la violencia de los años 70's y 80's. Finalmente, las personas mayores de 56 años que, dependiendo de su edad, pudieron haber presenciado varios periodos de violencia. La Tabla 3 muestra la distribución de los entrevistados por grupo de edad.

\section{Tabla 3. Distribución de entrevistas por grupo de edad de los entrevistados}

\begin{tabular}{|c|c|c|}
\hline Grupo de edad & Frecuencia & Promedio \\
\hline Menos de 18 años & 2 & $1,32 \%$ \\
\hline De 18 a 34 años & 71 & $46,71 \%$ \\
\hline De 35 a 55 años & 53 & $34,87 \%$ \\
\hline Más de 56 años & 26 & $17,11 \%$ \\
\hline Total general & 152 & $100 \%$ \\
\hline
\end{tabular}


En relación con el género, $48,7 \%$ de las personas entrevistadas se identificaron con el género femenino, mientras que el 51,3\% se identificó con el género masculino. La Tabla 4 muestra la distribución de los entrevistados por género.

\section{Tabla 4. Distribución de entrevistas POR gÉNero de los entreVistados}

\begin{tabular}{|c|c|c|}
\hline Género & Frecuencia & Promedio \\
\hline Femenino & 74 & $48,7 \%$ \\
\hline Masculino & 78 & $51,3 \%$ \\
\hline Total & 152 & $100 \%$ \\
\hline
\end{tabular}

Fuente: En Modo P. Elaboración propia

El lugar de procedencia de las personas entrevistadas se presenta por departamentos, excepto para Bogotá. Esto se justifica en la necesidad de resaltar a Bogotá como la capital de Colombia, imbuida en su propia dinámica social, política y económica que, incluso, ha llevado al Estado a generar instrumentos de política pública particular para esta ciudad con alcance de región. La Tabla 5 muestra la distribución de los entrevistados por lugar de procedencia.

\section{Tabla 5. Distribución de entrevistas por lugar de procedencia de los entrevistados}

\begin{tabular}{|l|c|c|}
\hline Departamento & Frecuencia & Promedio \\
\hline Bogotá & 44 & $28,9 \%$ \\
\hline Valle del Cauca & 33 & $21,7 \%$ \\
\hline Antioquia & 19 & $12,5 \%$ \\
\hline Cauca & 14 & $9,2 \%$ \\
\hline Nariño & 9 & $5,9 \%$ \\
\hline Santander & 6 & $3,9 \%$ \\
\hline Caldas & 6 & $3,9 \%$ \\
\hline Caquetá & 4 & $2,6 \%$ \\
\hline Bolívar & 3 & $2,0 \%$ \\
\hline Huila & 3 & $2,0 \%$ \\
\hline Nte. de Santander & 2 & $1,3 \%$ \\
\hline Quindio & 2 & $1,3 \%$ \\
\hline Cundinamarca & 2 & $1,3 \%$ \\
\hline Cesar & 1 & $0,7 \%$ \\
\hline Guainía & 1 & $0,7 \%$ \\
\hline Atlántico & 1 & $0,7 \%$ \\
\hline Guajira & 1 & $0,7 \%$ \\
\hline Risaralda & 152 & $0,7 \%$ \\
\hline Total general & $100 \%$ \\
\hline
\end{tabular}

Fuente: En Modo P. Elaboración propia 


\section{Resultados preliminares}

A pesar de que el proyecto adoptó una metodología de corte cualitativa para registrar las emociones de las personas en cada uno de los momentos del proceso final del acuerdo de paz, en este artículo me interesa analizar de manera preliminar las tendencias identificadas en los resultados obtenidos. Vale decir que, hacer un análisis de tendencias implica hacer una revisión cuantitativa de los resultados, en tanto se pretende evidenciar un patrón, dinámica o comportamiento de los datos (Castellanos et al., 2011). No obstante, el enfoque de la investigación sigue siendo cualitativo, pues la lectura de los datos que se presenta a continuación se hace desde un enfoque interpretativo (Tarrés, 2004).

Las emociones más frecuentes entre las personas entrevistadas en Quito fueron felicidad $(21,1 \%)$ y esperanza $(21,1 \%)$, que se encuentran asociadas a sentimientos como la alegría y el entusiasmo, pero también al optimismo, la motivación y la expectativa que generó la posibilidad de encontrar una salida pacífica al conflicto armado. No obstante, las emociones de indignación $(13,2 \%)$ e injusticia $(13,2 \%)$ se encuentran en segundo lugar, mostrando la otra cara de la moneda. Efectivamente, el contexto que medió las fechas de firma del acuerdo, la posterior refrendación y, finalmente, la aprobación y firma del acuerdo final se caracterizó por un momento de posiciones encontradas e incertidumbre $(9,9 \%)$, entre las personas que apoyaban el proceso de paz y aquellas que lo rechazaban. La Tabla 6 muestra los resultados generales por categoría emocional.

\section{TABla 6. Resultados generales POR CATEgoría EMOCIONAL}

\begin{tabular}{|l|c|c|}
\hline Categoría emocional & Frecuencia & Promedio \\
\hline Felicidad & 32 & $21,1 \%$ \\
\hline Esperanza & 32 & $21,1 \%$ \\
\hline Indignación & 20 & $13,2 \%$ \\
\hline Injusticia & 20 & $13,2 \%$ \\
\hline Incertidumbre & 15 & $9,9 \%$ \\
\hline Calma & 7 & $4,6 \%$ \\
\hline Satisfacción & 6 & $4,0 \%$ \\
\hline Euforia & 6 & $4,0 \%$ \\
\hline Bien & 5 & $3,3 \%$ \\
\hline Tristeza & 4 & $2,6 \%$ \\
\hline Satisfacción & 2 & $1,3 \%$ \\
\hline Conmoción & 1 & $0,7 \%$ \\
\hline Nostalgia & 1 & $0,7 \%$ \\
\hline Miedo & 1 & $0,7 \%$ \\
\hline Total & 152 & $100 \%$ \\
\hline
\end{tabular}

Fuente: En Modo P. Elaboración propia 
Sin duda, 2016 fue un año que quedó marcado en la memoria emocional de los colombianos, especialmente el último cuatrimestre. En septiembre se firmó el acuerdo de paz con las FARC, la guerrilla activa más vieja del país. En octubre se convocó al pueblo para refrendar el acuerdo, por una escasa diferencia se impuso el No en la consulta popular. Ese mismo mes, una semana después, el expresidente Juan Manuel Santos fue galardonado con el premio nobel de paz. Antes de terminar el mes, se elaboró un nuevo documento ajustado con las demandas de los grupos políticos que promovieron el voto por el No en el plebiscito. Finalmente, el 24 de noviembre se firmó el acuerdo para la terminación definitiva del conflicto en la ciudad de Bogotá, y empezaron las acciones para su implementación (S.A., 2016). El carrusel de emociones que marcó este proceso, así como la paulatina disminución de la felicidad y la esperanza, y el aumento de la indignación a medida que transcurría la implementación de los acuerdos, en los meses de diciembre, enero y febrero de 2017, se ve reflejada en la Tabla 7.

\section{TABLa 7. Distribución de LOS RESULTAdos POR MES Y AÑo}

\begin{tabular}{|l|c|c|c|c|c|c|c|c|c|}
\multirow{2}{*}{$\begin{array}{l}\text { Categoría } \\
\text { emocional }\end{array}$} & \multicolumn{7}{|c|}{$\mathbf{2 0 1 6}$} & \multicolumn{3}{|c|}{$\mathbf{2 0 1 7}$} & \multirow{2}{*}{ Total } \\
\cline { 2 - 9 } & $\mathbf{s e p t}$ & $\mathbf{o c t}$ & $\mathbf{n o v}$ & $\mathbf{d i c}$ & $\begin{array}{c}\text { Total } \\
\mathbf{2 0 1 6}\end{array}$ & $\mathbf{f e b}$ & $\mathbf{m a r}$ & $\begin{array}{c}\text { Total } \\
\mathbf{2 0 1 7}\end{array}$ & \\
\hline Felicidad & $15,1 \%$ & $35,0 \%$ & $0,0 \%$ & $12,5 \%$ & $21,7 \%$ & $16,7 \%$ & $12,5 \%$ & $14,3 \%$ & $21,1 \%$ \\
\hline Esperanza & $32,1 \%$ & $16,7 \%$ & $11,8 \%$ & $25,0 \%$ & $22,5 \%$ & $16,7 \%$ & $0,0 \%$ & $7,1 \%$ & $21,1 \%$ \\
\hline Indignación & $5,7 \%$ & $10,0 \%$ & $29,4 \%$ & $25,0 \%$ & $11,6 \%$ & $33,3 \%$ & $25,0 \%$ & $28,6 \%$ & $13,2 \%$ \\
\hline Injusticia & $11,3 \%$ & $11,7 \%$ & $23,5 \%$ & $12,5 \%$ & $13,0 \%$ & $0,0 \%$ & $25,0 \%$ & $14,3 \%$ & $13,2 \%$ \\
\hline Incertidumbre & $9,4 \%$ & $10,0 \%$ & $11,8 \%$ & $0,0 \%$ & $9,4 \%$ & $0,0 \%$ & $25,0 \%$ & $14,3 \%$ & $9,9 \%$ \\
\hline Satisfacción & $3,8 \%$ & $5,0 \%$ & $5,9 \%$ & $12,5 \%$ & $5,1 \%$ & $0,0 \%$ & $12,5 \%$ & $7,1 \%$ & $5,3 \%$ \\
\hline Calma & $3,8 \%$ & $6,7 \%$ & $5,9 \%$ & $0,0 \%$ & $5,1 \%$ & $0,0 \%$ & $0,0 \%$ & $0,0 \%$ & $4,6 \%$ \\
\hline Euforia & $7,5 \%$ & $3,3 \%$ & $0,0 \%$ & $0,0 \%$ & $4,3 \%$ & $0,0 \%$ & $0,0 \%$ & $0,0 \%$ & $3,9 \%$ \\
\hline Bien & $5,7 \%$ & $1,7 \%$ & $0,0 \%$ & $12,5 \%$ & $3,6 \%$ & $0,0 \%$ & $0,0 \%$ & $0,0 \%$ & $3,3 \%$ \\
\hline Tristeza & $3,8 \%$ & $0,0 \%$ & $5,9 \%$ & $0,0 \%$ & $2,2 \%$ & $16,7 \%$ & $0,0 \%$ & $7,1 \%$ & $2,6 \%$ \\
\hline Conmoción & $0,0 \%$ & $0,0 \%$ & $0,0 \%$ & $0,0 \%$ & $0,0 \%$ & $16,7 \%$ & $0,0 \%$ & $7,1 \%$ & $0,7 \%$ \\
\hline Miedo & $0,0 \%$ & $0,0 \%$ & $5,9 \%$ & $0,0 \%$ & $0,7 \%$ & $0,0 \%$ & $0,0 \%$ & $0,0 \%$ & $0,7 \%$ \\
\hline Nostalgia & $1,9 \%$ & $0,0 \%$ & $0,0 \%$ & $0,0 \%$ & $0,7 \%$ & $0,0 \%$ & $0,0 \%$ & $0,0 \%$ & $0,7 \%$ \\
\hline Total & $100 \%$ & $100 \%$ & $100 \%$ & $100 \%$ & $100 \%$ & $100 \%$ & $100 \%$ & $100 \%$ & $100 \%$ \\
\hline
\end{tabular}

Fuente: En Modo P. Elaboración propia 
A medida que aumenta la edad de las personas entrevistadas, las emociones se desplazan desde la felicidad hasta la indignación y la injusticia. Así, en la Tabla 8 se puede ver que las emociones predominantes en los relatos de las personas menores de 18 años y las personas entre 18 y 34 años son felicidad (22,5\% y $24,5 \%$ ) y esperanza (19,7\% y $26,4 \%)$. Mientras tanto, en las personas entre 35 y 55 años predomina la injusticia (23,1\%). Las personas mayores de 56 años se encuentran divididas entre felicidad $(50 \%)$ e indignación $(50 \%)$.

\section{TABLA 8. Distribución de los Resultados POR GRUPO DE EDA}

\begin{tabular}{|c|c|c|c|c|c|}
\hline $\begin{array}{l}\text { Categoría } \\
\text { emocional }\end{array}$ & $\begin{array}{l}\text { Menos de } 18 \\
\text { años }\end{array}$ & $\begin{array}{c}\text { De } 18 \text { a } 34 \\
\text { años }\end{array}$ & $\begin{array}{c}\text { De } 35 \text { a } 55 \\
\text { años }\end{array}$ & $\begin{array}{l}\text { Más de } 56 \\
\text { años }\end{array}$ & Total \\
\hline Felicidad & $22,5 \%$ & $24,5 \%$ & $7,7 \%$ & $50,0 \%$ & $21,1 \%$ \\
\hline Esperanza & $19,7 \%$ & $26,4 \%$ & $15,4 \%$ & $0,0 \%$ & $21,1 \%$ \\
\hline Indignación & $14,1 \%$ & $9,4 \%$ & $15,4 \%$ & $50,0 \%$ & $13,2 \%$ \\
\hline Injusticia & $9,9 \%$ & $13,2 \%$ & $23,1 \%$ & $0,0 \%$ & $13,2 \%$ \\
\hline Incertidumbre & $9,9 \%$ & $7,5 \%$ & $15,4 \%$ & $0,0 \%$ & $9,9 \%$ \\
\hline Satisfacción & $5,6 \%$ & $3,8 \%$ & $7,7 \%$ & $0,0 \%$ & $5,3 \%$ \\
\hline Calma & $4,2 \%$ & $5,7 \%$ & $3,8 \%$ & $0,0 \%$ & $4,6 \%$ \\
\hline Euforia & $4,2 \%$ & $1,9 \%$ & $7,7 \%$ & $0,0 \%$ & $3,9 \%$ \\
\hline Bien & $4,2 \%$ & $1,9 \%$ & $3,8 \%$ & $0,0 \%$ & $3,3 \%$ \\
\hline Tristeza & $2,8 \%$ & $3,8 \%$ & $0,0 \%$ & $0,0 \%$ & $2,6 \%$ \\
\hline Conmoción & $0,0 \%$ & $1,9 \%$ & $0,0 \%$ & $0,0 \%$ & $0,7 \%$ \\
\hline Miedo & $1,4 \%$ & $0,0 \%$ & $0,0 \%$ & $0,0 \%$ & $0,7 \%$ \\
\hline Nostalgia & $1,4 \%$ & $0,0 \%$ & $0,0 \%$ & $0,0 \%$ & $0,7 \%$ \\
\hline Total & $100 \%$ & $100 \%$ & $100 \%$ & $100 \%$ & $100 \%$ \\
\hline
\end{tabular}

Fuente: En Modo P. Elaboración propia

La tendencia general de los resultados, en los que predominan las emociones de felicidad $(21,1 \%)$, esperanza $(21,1 \%)$, indignación $(13,2 \%)$, injusticia $(13,2 \%)$ e incertidumbre $(9,9 \%)$, se mantiene al momento de hacer el análisis comparado por género. No obstante, resulta interesante que, en el caso de las personas de género masculino la emoción que más se refleja en los relatos sea la felicidad $(24,4 \%)$, mientras que en las personas de género femenino sea la esperanza (23\%). Esta relación inversa puede estar justificada en la forma cómo cada uno de los géneros expresan las emocionalidades, aunque también podría justificarse en los picos de emocionalidad causados por cada uno de los acontecimientos del proceso final del acuerdo de paz. La Tabla 9 muestra los resultados distribuidos por género. 


\section{TABla 9. Distribución de los resultados por género}

\begin{tabular}{|l|c|c|c|}
\hline Categoría emocional & Femenino & Masculino & Total \\
\hline Felicidad & $17,6 \%$ & $24,4 \%$ & $21,1 \%$ \\
\hline Esperanza & $23,0 \%$ & $19,2 \%$ & $21,1 \%$ \\
\hline Indignación & $16,2 \%$ & $10,3 \%$ & $13,2 \%$ \\
\hline Injusticia & $9,5 \%$ & $16,7 \%$ & $13,2 \%$ \\
\hline Incertidumbre & $13,5 \%$ & $6,4 \%$ & $9,9 \%$ \\
\hline Satisfacción & $2,7 \%$ & $7,7 \%$ & $5,3 \%$ \\
\hline Calma & $4,1 \%$ & $5,1 \%$ & $4,6 \%$ \\
\hline Euforia & $4,1 \%$ & $3,8 \%$ & $3,9 \%$ \\
\hline Bien & $4,1 \%$ & $2,6 \%$ & $3,3 \%$ \\
\hline Tristeza & $4,1 \%$ & $1,3 \%$ & $2,6 \%$ \\
\hline Conmoción & $1,4 \%$ & $0,0 \%$ & $0,7 \%$ \\
\hline Miedo & $0,0 \%$ & $1,3 \%$ & $0,7 \%$ \\
\hline Nostalgia & $100 \%$ & $100 \%$ \\
\hline Total & & & \\
\hline
\end{tabular}

Fuente: En Modo P. Elaboración propia

Finalmente, se presentan los resultados desagregados por el lugar de origen de los entrevistados. Como se puede apreciar en la tabla 10, existe una interesante contradicción entre las tres principales regiones del país. Mientras que en los relatos de las personas cuyo lugar de origen es Bogotá predominan las emociones de esperanza (20,5\%) y felicidad (29,5\%), en los relatos de las personas de Antioquia (cuya capital es Medellín) y Valle del Cauca (con Cali como ciudad capital) predominan la indignación $(21,1 \%$ y $27,3 \%$ ) y la injusticia $(26,3 \%$ y $15,2 \%)$. Dos razones de orden histórico-político podrían justificar estos resultados. La primera, obedece a la dinámica centro-periferia que ha definido históricamente la rivalidad entre los centros de poder nacional y regional en los países latinoamericanos. En el caso particular de Colombia, esto también se ve reflejado la relación directa o indirecta que tienen las personas con el conflicto. La segunda, razón obedece a la rivalidad política entre los expresidentes Álvaro Uribe, oriundo del departamento de Antioquia, y Juan Manuel Santos de Bogotá. Para el caso de la refrendación de los acuerdos de paz mediante plebiscito, esta rivalidad se tradujo en posturas a favor (campaña del Sí, liderada por Juan Manuel Santos) y en contra (campaña del No, liderada por Álvaro Uribe). Discusión 


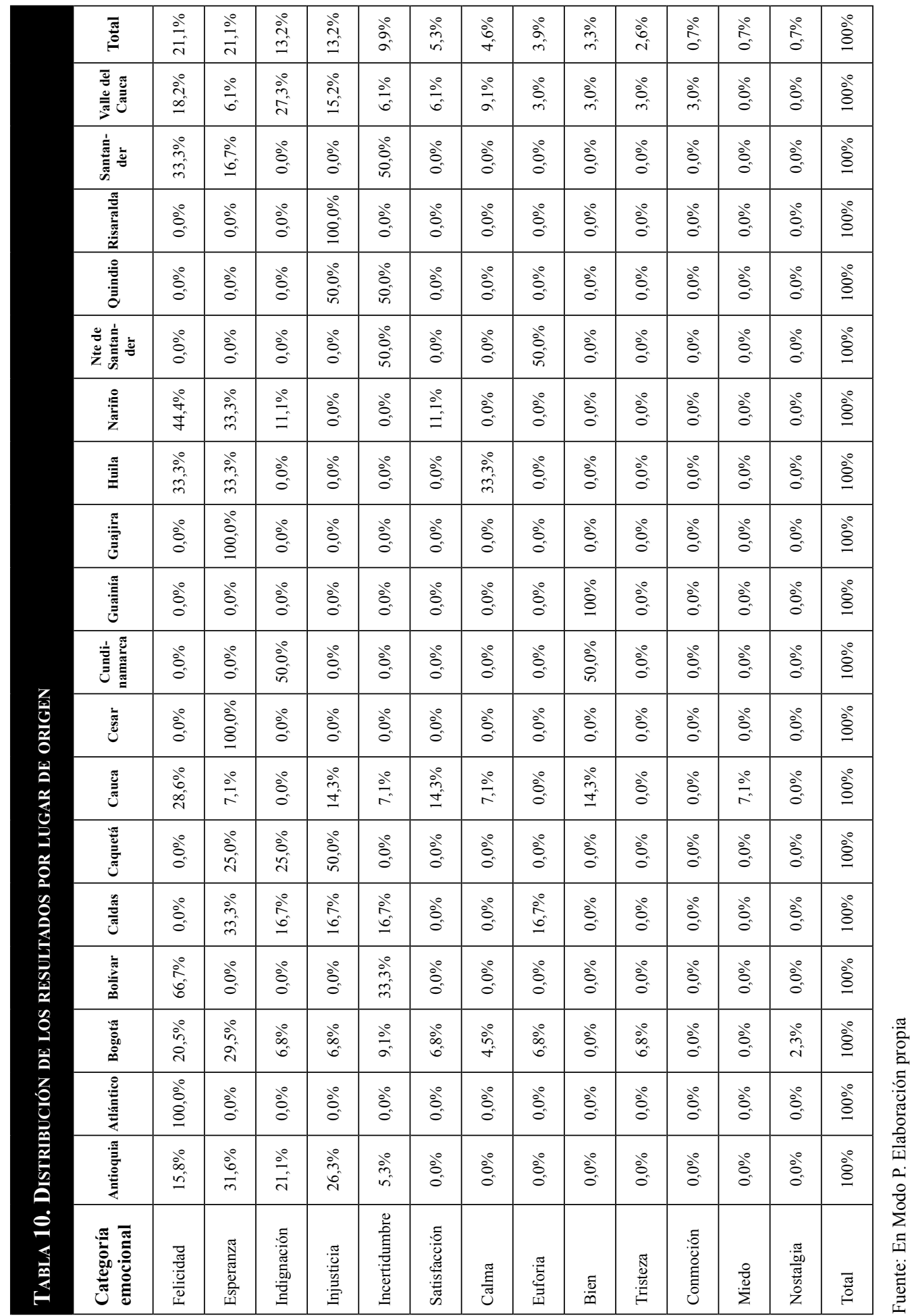


A partir de una lectura transversal de los datos se podría afirmar que, a pesar de que los relatos de las personas entrevistadas están encauzados hacia la construcción de un relato particular del conflicto armado en Colombia, las emociones que generó el proceso de paz con las FARC y las expectativas que abre la posibilidad del postconflicto, explorar los datos al nivel que se ha propuesto en este artículo revela cómo los sentimientos construyen realidad social y cómo, a partir de ellos, las personas construyen el significado social de sus instituciones.

Efectivamente, el análisis de los resultados preliminares permite identificar algunos patrones en la evolución del imaginario social de paz en Colombia asociado a los lugares de la memoria en el encuentro con la historia del conflicto de las personas. Adicionalmente, estas tendencias permiten construir una percepción común del conjunto social en cada uno de los momentos del proceso, permitiendo, a su vez, explorar los intersticios de encuentro entre las esferas de la subjetividad y la materialidad de las personas (Anderson, 2009; citado por Lara y Enciso, 2013).

Los resultados reflejan a primera vista, la segmentación de la población colombiana residente en Quito alrededor de dos ideas contradictorias del acuerdo de paz. En primer lugar, la postura adoptada por el gobierno nacional desde el año 2012, que apostaba por una salida negociada del conflicto. La segunda postura estaba representada por los líderes políticos opositores al gobierno de turno, que veían en el acuerdo de paz con la FARC la rendición del Estado ante una de las fuerzas armadas más temibles y controvertidas de la historia latinoamericana.

\footnotetext{
"Bueno mucha mucha alegría por salir por encontrar una salida negociada al conflicto se va acabar una un episodio trágico en la historia nacional que ha cobrado muchas muchas víctimas mucho daño que terminan desplazados terminan con división interna una buena noticia para el país la finalización de la guerra" (relato de persona de género masculino de 26 años, lugar de procedencia: Bolívar. Fecha de grabación: 26 de septiembre de 2016)

"Ceo que se va a seguir fortaleciendo se va a seguir fortaleciendo la ELN y eso pero de todas maneras va a tocar hacerlo y poco a poco yo creo que la gente va reaccionando yo conozco mucha gente que ha terminado con muchas de sus familias y es muy doloroso claro yo no viví eso porque yo estoy aquí desde hace muchos años pero conozco mucha gente que ha sufrido mucho por eso y pienso que a pesar de todo hay que votar por el sí muy alterada en realidad porque no es una cosa fácil de digerir mejor dicho yo pienso pero no lo digiero me duele muchísimo todo lo que está pasando y todo lo que la gente ha sufrido" (relato de persona de género femenino de 81 años, lugar de procedencia: Bogotá. Fecha de grabación: 18 de septiembre de 2016).
}

Traducido al lenguaje de las atmósferas afectivas de Anderson (2009), en cada uno de los momentos del proceso final del acuerdo de paz, se pueden vislumbrar vestigios de estos momentos en donde los afectos y las afecciones (Lerner, 1998) afectan la realidad social. De esta forma, la primera atmósfera que se logra percibir está dominada por la felicidad y la esperanza, como reflejo de la alegría, la dicha, el entusiasmo, la expectativa, el optimismo y la motivación que causa en las personas la idea de un país en paz. La segunda atmósfera que salta a la vista, producto del análisis de los resultados preliminares, está relacionada con las emociones de indignación e injusticia relacionadas, a su vez, con la furia, el enojo, la frustración, el enfado, la insatisfacción, la impotencia y el pesimismo que causó la idea de entregar el país a las FARC, asociada a la derrota de las fuerzas del Estado en el campo de batalla (Salas, 2017).

La materialidad de estas manifestaciones subjetivas alcanzó su mayor punto el 2 de octubre de 2018, cuando el pueblo colombiano fue llamado a las urnas para decidir si aceptaba o no el acuerdo de paz. Una de las premisas del giro afectivo es que aquello que se siente afecta la materialidad de la vida cotidiana, apelando para ello a la emocionalización de la vida pública y de los sistemas y subsistemas que la conforman. Esto fue lo que denominó Corinne Squire (2001) como "sociedades afectivas". Ese día, ambas atmósferas se encontraron, generando un ambiente generalizado por la expectativa y la incertidumbre. Los resultados ese día son la viva 
evidencia de esto. Al final de la jornada, las emociones se contrapusieron. Las personas que estaban imbuidas en la primara atmósfera pasaron repentinamente a la segunda atmósfera, y viceversa.

"A ver es algo muy lindo lo que el gobierno está haciendo referente a la paz pero más yo no creo en ella voté por el sí porque es algo que queremos que nuestro país surja pero que es lo que está haciendo ahorita la guerrilla de las FARC están entregando las armas al ELN por qué no negociaron con las dos a la vez porque quieren dejar armas guardadas las FARC no es justifico de que ellos guarden armas para qué siempre y cuando van hacer la paz entreguen todas las armas pero no se justifica de que ese grupo tenga armas" (relato de persona de género masculino de 49 años, lugar de procedencia: Valle del Cauca. Fecha de grabación: 2 de octubre de 2016).

A pesar de este movimiento brusco que se ve reflejado en los datos, especialmente en la lectura longitudinal de los resultados distribuidos por mes y años (ver Tabla 11), a partir de noviembre surge una tercera atmósfera predominada por la incertidumbre, como el reflejo de la duda, la preocupación y la intriga que causó en las personas los turbulentos acontecimientos del mes de octubre.

\section{TABla 11. Distribución de los Resultados por mes y año (LONGitudinal)}

\begin{tabular}{|c|c|c|c|c|c|c|c|c|c|}
\hline \multirow{2}{*}{$\begin{array}{l}\text { Categoría } \\
\text { emocional }\end{array}$} & & \multicolumn{4}{|c|}{2016} & \multirow[b]{2}{*}{ feb } & \multicolumn{2}{|c|}{2017} & \multirow{2}{*}{ Total } \\
\hline & sept & oct & nov & dic & $\begin{array}{l}\text { Total } \\
2016 \\
\end{array}$ & & $\operatorname{mar}$ & $\begin{array}{l}\text { Total } \\
2017 \\
\end{array}$ & \\
\hline Felicidad & $25,0 \%$ & $65,6 \%$ & $0,0 \%$ & $3,1 \%$ & $93,8 \%$ & $3,1 \%$ & $3,1 \%$ & $6,3 \%$ & $100 \%$ \\
\hline Esperanza & $53,1 \%$ & $31,3 \%$ & $6,3 \%$ & $6,3 \%$ & $96,9 \%$ & $3,1 \%$ & $0,0 \%$ & $3,1 \%$ & $100 \%$ \\
\hline Indignación & $15,0 \%$ & $30,0 \%$ & $25,0 \%$ & $10,0 \%$ & $80,0 \%$ & $10,0 \%$ & $10,0 \%$ & $20,0 \%$ & $100 \%$ \\
\hline Injusticia & $30,0 \%$ & $35,0 \%$ & $20,0 \%$ & $5,0 \%$ & $90,0 \%$ & $0,0 \%$ & $10,0 \%$ & $10,0 \%$ & $100 \%$ \\
\hline Incertidumbre & $33,3 \%$ & $40,0 \%$ & $13,3 \%$ & $0,0 \%$ & $86,7 \%$ & $0,0 \%$ & $13,3 \%$ & $13,3 \%$ & $100 \%$ \\
\hline Satisfacción & $25,0 \%$ & $37,5 \%$ & $12,5 \%$ & $12,5 \%$ & $87,5 \%$ & $0,0 \%$ & $12,5 \%$ & $12,5 \%$ & $100 \%$ \\
\hline Calma & $28,6 \%$ & $57,1 \%$ & $14,3 \%$ & $0,0 \%$ & $100 \%$ & $0,0 \%$ & $0,0 \%$ & $0,0 \%$ & $100 \%$ \\
\hline Euforia & $66,7 \%$ & $33,3 \%$ & $0,0 \%$ & $0,0 \%$ & $100 \%$ & $0,0 \%$ & $0,0 \%$ & $0,0 \%$ & $100 \%$ \\
\hline Bien & $60,0 \%$ & $20,0 \%$ & $0,0 \%$ & $20,0 \%$ & $100 \%$ & $0,0 \%$ & $0,0 \%$ & $0,0 \%$ & $100 \%$ \\
\hline Tristeza & $50,0 \%$ & $0,0 \%$ & $25,0 \%$ & $0,0 \%$ & $75,0 \%$ & $25,0 \%$ & $0,0 \%$ & $25,0 \%$ & $100 \%$ \\
\hline Conmoción & $0,0 \%$ & $0,0 \%$ & $0,0 \%$ & $0,0 \%$ & $0,0 \%$ & $100 \%$ & $0,0 \%$ & $100 \%$ & $100 \%$ \\
\hline Miedo & $0,0 \%$ & $0,0 \%$ & $100,0 \%$ & $0,0 \%$ & $100,0 \%$ & $0,0 \%$ & $0,0 \%$ & $0,0 \%$ & $100 \%$ \\
\hline Nostalgia & $100 \%$ & $0,0 \%$ & $0,0 \%$ & $0,0 \%$ & $100 \%$ & $0,0 \%$ & $0,0 \%$ & $0,0 \%$ & $100 \%$ \\
\hline
\end{tabular}

Fuente: En Modo P. Elaboración propia 
Esta tercera atmósfera se configura a partir del escepticismo que generó los mecanismos de implementación de los acuerdos que, en su contenido transmiten la complejidad del proceso, pero, al mismo tiempo, desconocen la complejidad del contexto social y político del país. Antivalores asociados a la corrupción, la inmoralidad y la deshonestidad son comúnmente referidos en los relatos las personas entrevistadas para justificar la incertidumbre que generó la implementación del acuerdo final de paz, las condiciones materiales para el retorno a Colombia, en el caso de la población refugiada, y la expectativa de cambio social a partir de la entrada en el juego político de un nuevo actor con ideas de izquierda (Partido FARC) a partir de los comicios electorales del año 2018.

"Incertidumbre angustia eso que va a pasar con el resto de grupos guerrilleros que no solo son las FARC si gana el no que va hacer toda esa cantidad de guerrilleros fuera son personas que son se criaron se educaron a ser guerrilla a matar hacer cosas malas nada productivo para Colombia que va hacer toda esa cantidad de gente afuera es mi pregunta que va a ser la vida de todos ellos y de todo el pueblo" (relato de persona de género femenino de 28 años, lugar de procedencia: Cauca. Fecha de grabación: 2 de octubre de 2016).

No obstante, estos resultados generales, queda la enorme tarea de realizar un análisis más profundo del contenido de los relatos que permita dar forma a las tres atmósferas afectivas identificadas en la lectura propuesta en este artículo, para identificar mejor la conexión existente entre las esferas de la subjetividad y la materialidad de la vida pública. 


\section{Agradecimientos}

$\mathrm{Al}$ equipo de trabajo y todo el grupo de colaboradores del proyecto En Modo P, especialmente a Susana Wappenstein, Olga Lucía Lozano, Laura López, Diana Tejedor, Laura Lozano y Carlos Flores.

\section{Bibliografía}

Anderson, Ben. 2009, “Affective atmosphere”, en: Emotion, Space an Society, 2(22), pp. 77-81.

Arboleda, Raúl (14 de octubre de 2016), "La banda sonora de la violencia en Colombia", en: El País, recuperado de: http://elpaissemanal.elpais.com/documentos/labandasonoracolombia/

Castellanos, Oscar et al., 2011, Análisis de tendencias: de la información a la innovación, Universidad Nacional de Colombia, Bogotá, recuperado de: http://bdigital.unal.edu.co/3564/1/ANALISIS_DE_TENDENCIAS_ MAYO_7.pdf

Codhes. 2018, "Exilio, migración forzada, construcción de paz en Colombia", Consultoría para los Derechos Humanos y el Desplazamiento, en: Iranzo-Dosdad, Ángela et al. (Comp.) Entre la guerra y la paz: los lugares de la diáspora colombiana, Universidad de los Andes - Pontificia Universidad Javeriana, Bogotá.

El País (24 de noviembre de 2016), "Gobierno y las FARC firmaron acuerdo de paz en Bogotá", en: El País, recuperado de: https://www.elpais.com.co/elpais/colombia/proceso-paz/noticias/gobierno-y-farc-firmannuevo-acuerdo-paz-bogota-0

Hardt, Michel. 2007, "Foreword: what affects are good for", en: Clough, Patricia y Halley, Jean (Eds.). The Affective Turn, Durkham, Duke University Press, London.

Lara, Alí y Enciso-Domínguez, Giazú. 2013, "El Giro Afectivo”, en: Athenea Digital, 13(3), pp. 101-119.

León, Juanita. (20 de enero de 2017), "El nuevo proyecto de nuestra Olga Lucía", en: Lasillavacia.com., recuperado de: $\mathrm{http}: / /$ lasillavacia.com/historia/elnuevoproyectodenuestraolgalucia59450

Lerner, Hugo. 1998, “Afectos, afecciones y afectaciones”, en: Psicoanalisis APdeBA XX (3), pp. 683-704.

Macón, Cecilia. 2013, "Sentimus ergo sumus. El surgimiento del "giro afectivo" y su impacto en la filosofía política", en: Revista Latinoamericana de Filosofia Política II (6), pp. 1-32.

Oficina del Alto Comisionado Para La Paz - OACP. 2016, "El Acuerdo Final de Paz", disponible en: http://www. altocomisionadoparalapaz.gov.co/herramientas/Documents/Nuevo_enterese_version_6_Sep_final_web.pdf

Ortega, Hernando y Riaño-Alcalá, Pilar. 2007, "El refugio desde la experiencia de la población", en: VillaMartínez, Marta y Riaño-Alcalá, Pilar (Coord.). Migración forzada de colombianos. Colombia, Ecuador, Canadá, FLACSO sede Ecuador-Corporación Región-UBC, Quito-Medellín, pp. 62-91.

Rincón-Suárez, Liz. 2018, “Todos vuelve: ciudadanías posnacionales e imaginaciones que retornan”, en: IranzoDosdad, Ángela y Edson-Louidor, Wooldy (Comp.) Entre la guerra y la paz: los lugares de la diáspora colombiana, Universidad de los Andes - Pontificia Universidad Javeriana, Bogotá.

Salas, Jesús David (6 de junio de 2017), "El pulso de la paz en Colombia", en: Imaginación o Barbarie, Boletín mensual de opinión de la Red Iberoamericana de Investigación en Imaginarios y Representaciones (4), pp. 50-55, recuperado de: https://www.academia.edu/33432957/Imaginaci\%C3\%B3n_o_barbarie_n.o_4

16 de diciembre de 2017, "Sobre la cuestionable estrategia de campaña del No", en: Imaginación o Barbarie, Boletín mensual de opinión de la Red Iberoamericana de Investigación en Imaginarios y Representaciones (10), pp. 296-299, recuperado de: https://imaginariosyrepresentaciones.files.wordpress.com/2017/12/ recopilatorioespecial_vf1.pdf

Sánchez-Mesa, Domingo, et al. 2016, “Transmedia (Storytelling?): a polyphonic critical review”, en: Artnodes (18), pp. 8-17, recuperado de: http://artnodes.uoc.edu

Squire, Corinne. 2001, "The public life of emotions", en: International Journal of Critical Psychology, 1, pp. 2738, recuperado de: http//www.lwbooks.co.uk/journals.html

Tarrés, María Luisa. 2004, "Lo cualitativo como tradición", en: Observar, Escuchar y Comprender: sobre la tradición cualitativa en la investigación social, FLACSO-COLMEX, México.

Thrift, Nigel. 2008, Non-representational theory: space, politics, affect, Routledge, London - New York. 